\title{
Amplification of phytochrome induced morphogenesis in plants by the cryptic red light signal (CRS)
}

Lakshmi Nimmagadda* and Guruprasad Kadur Narayanaswamy

School of Life Sciences; Devi Ahilya University; Indore, India

Key words: ascorbic acid, cryptic red light signal, glycine max, phytochrome, ultraviolet-B

The regulation of endogenous levels of ascorbic acid in soybean by far-red absorbing form of phytochrome (Pfr) and by cryptic red light signal (CRS) was studied. Cryptic red light signal is produced by red light pre-irradiation of a photoreceptor other than far-red absorbing form of phytochrome (Pfr) and CRS amplifies the action of phytochrome. The endogenous level of ascorbic acid levels enhanced by phytochrome was amplified by CRS. The lifetime of CRS was from 0 to $2 \mathrm{~h}$ and the peak of enhancement of ascorbic acid due to CRS was between 16 to $24 \mathrm{~h}$ of dark incubation after the end of the treatment. CRS was found to be ineffective on UV-B enhanced endogenous levels of ascorbic acid.

The phytochrome mediated morphogenesis involves the conversion of $\operatorname{Pr}$ [red absorbing form] to Pfr [far-red absorbing form] and the magnitude of the response is dependent on $\mathrm{Pfr} / \mathrm{P}$ tot ratio established at the end of the irradiation. ${ }^{1}$ In broom Sorghum anthocyanin synthesis induced by red light $\left[R_{1}\right]$ is reversible with far-red light. But a second red pulse $\left[R_{2}\right]$ given after the reversal resulted in increased anthocyanin production compared to the first pulse $\left[R_{1}\right]$. When the red pulse was repeatedly given after every reversal with far-red, the anthocyanin production increased proportionately to the number of previously given pulses. ${ }^{2}$ Thus red pre-treatment induced a change in the cellular physiological state or change in content of a relevant substance[s] which is designated as Cryptic Red Light Signal [CRS] associated with red signal transduction. $^{2}$ CRS was first characterized in detail in Broom Sorghum as Pfr amplifying signal produced by red pre-irradiation. CRS is inactive in the absence of Pfr but enhances the action of Pfr. CRS escapes reversal when the plants are exposed to far-red and is

*Correspondence to: Lakshmi Nimmagadda; School of Life Sciences; Vigyan Bhawan; Devi Ahilya University; Takshashila Campus; Khandwa Road; Indore 452001 M.P. India; Email: lakshmi_n26@hotmail.com

Submitted: 03/04/09; Accepted: 03/06/09

Previously published online as a Plant Signaling \& Behavior E-publication: http://www.landesbioscience.com/journals/psb/article/8346

Addendum to: Nimmagadda L, Kadur NG. Cryptic red light signal regulates ascorbic acid in soybean. J Plant Physiol 2009; 166:329-32; PMID: 18786744; DOI: 10.1016/i.jplph.2008.06.006. probably produced by a different species of phytochrome, distinct from the conventional reversible phytochrome. ${ }^{3}$

We have investigated whether CRS influences other phytochrome regulated processes in plants in addition to anthocyanin synthesis. We chose another process, the synthesis of endogenous ascorbic acid, which is also regulated by conventional phytochrome. ${ }^{4}$ In soybean, the endogenous level of ascorbic acid is enhanced by conventional far-red reversible form of phytochrome. In addition, an independent UV-B photoreceptor [non reversible with far-red light] also enhances the endogenous synthesis of ascorbic acid in soybean. By using repeated pulses of red light, we have demonstrated that the Cryptic Red Signal is operative in soybean also and it amplifies the red light induced enhancement in the level of ascorbic acid. That CRS is active only in the presence of $\mathrm{Pfr}$ is demonstrated by the fact that pre-irradiation with red light is ineffective in amplifying UV-B induced enhancement of ascorbic acid levels. A similar observation on UV-B induced anthocyanin synthesis has been made in Broom Sorghum. ${ }^{2}$ A separate UV-B photoreceptor independent of phytochrome operates in the plants. ${ }^{5}$ Although CRS is presumably produced by pre-irradiation with red light, it does not enhance UV-B induced anthocyanin synthesis or ascorbic acid synthesis in the absence of formation of Pfr by the second red pulse.

The life-time of CRS was determined as $6 \mathrm{~h}$ in $20^{\circ} \mathrm{C}$ and $3 \mathrm{~h}$ in $24^{\circ} \mathrm{C}$ grown seedlings of Broom Sorghum with reference to anthocyanin synthesis. ${ }^{2}$ The life-time of CRS determined in soybean seedlings grown at $25^{\circ} \mathrm{C}$ was upto $1 \mathrm{~h}^{6}$ Since growing seedlings at a low temperature enhanced the effectiveness of CRS in Broom Sorghum, it was concluded that low temperature may either extend the lifetime of CRS or generate higher amount of CRS. ${ }^{2}$ Although the exact nature of CRS is yet to be analyzed, work in our laboratory has established the universal nature of this signal and evidences have been obtained for CRS effect in promoting red light induced hypocotyls inhibition in Cucumber seedlings and also red light induced synthesis of betacyanins in Amaranthus seedlings (submitted for publication). 


\section{Ascorbic acid regulation by CRS}

\section{References}

1. Mancinelli AL. The physiology of phytochrome action. In: Photomorphogenesis in Plants. Kondrick RE, Khonenburg GHM, (eds). Kluwer Academic Publishers, Dodrecht, the Netherlands 211-70.

2. Shichijo C, Hashimoto T. A red light signal distinct from the far-red-absorbing form of phytochrome in anthocyanin induction of Sorghum bicolor. J Photochem Photobiol B 1997; 38:70-5.

3. Hashimoto T, Shichijo C. Amplification of phytochrome-induced photomorphogenesis by red light pre-irradiation and a cryptic red-light signal production. Trends Photochem Photobiol 1999; 6:17-27.

4. Schopfer P. The control by phytochrome of the contents of ascorbic acid and dehydroascorbic acid in the mustard seedlings (Sinapis alba L). Planta 1966; 69:158-77.

5. Yatsuhashi H, Hashimoto T, Shimizu S. Ultraviolet action spectrum for anthocyanin formation in broom sorghum first internodes. Plant Physiol 1982; 70:735-41.

6. Nimmagadda L, Kadur NG. Cryptic red light signal regulates ascorbic acid in soybean J Plant Physiol 2009; 166:329-32. 